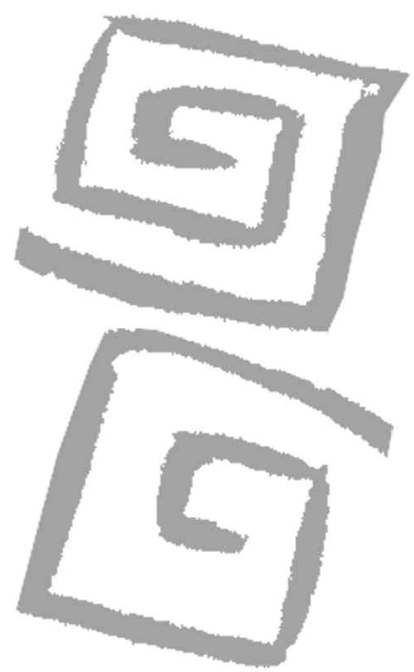

\title{
La lucha contra la poliomielitis: una alianza médico-social, Buenos Aires, 1943
}

\author{
The fight against polio: a social-medical alliance, \\ Buenos Aires, 1943
}

${ }^{1}$ Licenciada en Terapia Ocupacional. Magíster en Diseño y Gestión de Políticas Sociales. Doctoranda en Ciencias Sociales. Profesional, Hospital Interdisciplinario Psicoasistencial José Tiburcio Borda. Docente, Universidad de Buenos Aires, Argentina. danitestu@yahoo.com.ar
Testa, Daniela Edelvis ${ }^{1}$

RESUMEN El presente artículo analiza el surgimiento de una de las organizaciones voluntarias de asistencia social que tuvo una activa participación en la atención sanitaria de la poliomielitis en Argentina: la Asociación para la Lucha contra la Parálisis Infantil. Esta institución fue conformada en la ciudad de Buenos Aires, en 1943, por un grupo de mujeres de sectores sociales altos y medios. En un contexto de incertidumbre biomédica la entidad movilizó recursos materiales y simbólicos para dar respuesta al problema de la rehabilitación de las afecciones físicas permanentes que la enfermedad provocaba en sus víctimas. Utilizando como fuente las memorias institucionales de la organización, se muestra cómo médicos y filántropas constituyeron una alianza médico-social y desarrollaron el interés fundamental de practicar una modalidad de tratamiento -en un contexto donde todo estaba por hacerse- sostenidos en un andamiaje que amalgamaba ideas cristianas con innovaciones de la medicina y la rehabilitación.

PALABRAS CLAVE Poliomielitis; Médicos; Asistencia Social; Beneficencia; Rehabilitación.

ABSTRACT The present article analyzes the emergence of a volunteer-based social assistance organization that played an active role in the health care provided for poliomyelitis in Argentina: Asociación para la Lucha contra la Parálisis Infantil (Association for the Fight against Child Paralysis). This institution was created in Buenos Aires in 1943 by a group of women from upper and middle class social sectors. In a context of biomedical uncertainty, the organization mobilized material and symbolic resources to respond to the need for rehabilitation of the permanent physical conditions the disease provoked in its victims. Using as a source the institutional memories of the organization, the article demonstrates how doctors and philanthropists formed a social-medical alliance and developed a fundamental interest in practicing a form of treatment sustained in a framework that united Christian ideas with medical and rehabilitative innovations.

KEY WORDS Poliomyelitis; Physicians; Social Assistance; Beneficence; Rehabilitation. 


\section{INTRODUCCIÓN}

La poliomielitis es una enfermedad infectocontagiosa que afectó a gran cantidad de personas durante la primera mitad del siglo XX a través de la aparición de epidemias a nivel mundial. Los sucesivos y graves brotes de la enfermedad significaron un cambio en su forma de comportamiento y modificaron su estatus de enfermedad esporádica y endémica para ser percibida como un problema sanitario relevante. No obstante su previa existencia, fue recién en este período cuando pasó a ser considerada como una preocupación y causa de políticas sanitarias por parte de los Estados. Este cambio en el modo de ser contemplada no se debió solo al incremento en el número de casos o a la mortalidad que era capaz de provocar en casos severos, sino a la cantidad de personas que sobrevivían con secuelas y capacidades funcionales notablemente disminuidas (1-3).

Se han identificado tres agentes etiológicos serotipos de poliovirus. Los tres son extremadamente virulentos y producen los mismos síntomas de la enfermedad. El ser humano es el único reservorio y la infección se transmite de persona a persona por vía fecal-oral. No existe tratamiento específico para esta enfermedad y las medidas de sostén para conservar la vida, preservando las funciones vitales, constituyen la única forma de atención médica en la fase aguda.

La poliomielitis ha sido durante siglos una importante causa de invalidez y de muerte en la infancia hasta el advenimiento de la vacuna, a mediados de la década de 1950. Actualmente, las medidas preventivas se centran en la aplicación de la vacuna. Otras medidas incluyen la sensibilización y captación oportuna de casos, la educación a la población sobre el modo de transmisión y el cumplimiento del esquema de vacunación. Las medidas de control consisten en la notificación obligatoria a las autoridades de salud, así como la vacunación según esquema regular. La confirmación diagnóstica se realiza con datos clínicos, epidemiológicos y de laboratorio.

En la región de las Américas, la Comisión Internacional para la Certificación de la Erradicación de la Poliomielitis certificó la interrupción de la circulación de poliovirus salvaje en el año 1994. La mortalidad total producida por la poliomielitis paralítica en los momentos en que no se disponía de recursos tecnológicos avanzados para la respiración asistida (pulmotores, camas oscilantes) era del $5 \%$ al $10 \%$. Nótese que esta cifra no puede considerarse demasiado elevada. No obstante, lo que preocupaba era la cantidad de niños afectados con secuelas de la enfermedad y los trastornos aparejados, sumado al alto costo económico de los prolongados tratamientos de recuperación posterior que requerían el uso de aparatos especiales (ortesis, bastones, sillas de ruedas, respiradores).

Aunque los brotes de poliomielitis en la Argentina existieron desde 1906, el reconocimiento de su carácter epidémico data de 1936, año en el que el pediatra Juan Garrahan (4) consignó la existencia de un brote en la ciudad de Buenos Aires, criterio que fue compartido por otros médicos de la época (3). En consecuencia a tal reconocimiento fue en 1936 cuando se dio un primer paso hacia el control y prevención del contagio a través de la sanción de la Ley $\mathrm{N}^{\circ} 12.317$, que hacía obligatoria la denuncia de las enfermedades infecciosas trasmisibles ante las autoridades oficiales. De este modo, se comenzaron a elaborar los primeros registros estadísticos y se pudieron recabar datos empíricos para el estudio de la enfermedad en el país (4). De acuerdo a las tasas de incidencia de la enfermedad cada mil habitantes, las tres epidemias de mayor importancia de nuestro país fueron las correspondientes a los años 1936, (con una tasa de incidencia de 10 por mil), 1953 (14 por mil) y 1956 (33 por mil).

En Argentina, esta enfermedad afectó tanto a grupos sociales acomodados como populares de Buenos Aires y desafió la capacidad de la medicina y las prevenciones de las medidas higiénicas. La presencia de los enfermos crónicos, la imagen de "legión de niños lisiados" (a) (5 p.22) y la carga social que ello significaría en el futuro planteaban ansiedades amenazantes que se respiraban en el clima urbano de la época y que requería de urgentes soluciones $(5,6)$.

Entre las acciones cotidianas para evitar el contagio que han quedado en el recuerdo de los porteños están la utilización de las pastillas de alcanfor en la ropa, la limpieza de las veredas y calles realizada por los mismos vecinos, los árboles y cordones de las veredas blanqueados con cal, las fumigaciones aéreas con gamezzane y los 
traslados de los niños sanos hacia el campo; indicadores del temor y desasosiego frente a una enfermedad contra la cual no había aún soluciones efectivas ni definitivas.

En un contexto de incertidumbre biomédica -que persistiría hasta la aparición de la vacuna antipoliomielítica en $1954(6,7)$ - ante el problema de las secuelas de una enfermedad que no mataba pero dejaba a la vista grados de invalidez irreversibles, una iniciativa femenina inspirada en ideas filantrópicas constituyó, en 1943, en la ciudad de Buenos Aires, la Asociación para la Lucha contra la Parálisis Infantil (ALPI), entidad que canalizó sus esfuerzos en la necesidad de encontrar soluciones a los efectos posteriores de la polio.

Desde ese ámbito, estas mujeres se involucraron activamente y de forma pública con el problema y sus intervenciones sociales avanzaron gradualmente hacia una mayor complejidad, variedad y alcance. Entre ellas se incluyeron un conjunto de medidas de tipo médico-social tendientes a favorecer y poner en práctica las novedades de la medicina en el campo de la poliomielitis. Para ello, no solo brindaron una estructura institucional asistencial, sino que destinaron esfuerzos a fomentar la continua formación del equipo de profesionales y su participación en intercambios científicos. El otorgamiento de becas de perfeccionamiento, la continua participación de representantes de la entidad en eventos científicos internacionales y nacionales, la divulgación de materiales educativos y de prevención sobre el contagio dirigidos a la población en general demuestran el interés en apoyar el desarrollo del conocimiento en el área y en posicionarse como una entidad de referencia en el tema (8 p.5).

De acuerdo con lo señalado por Donna Guy, en Argentina, desde las damas de la Sociedad de Beneficencia hasta otras variadas organizaciones femeninas tuvieron una amplia gama de motivaciones y concepciones acerca de la beneficencia que significaron un valioso aporte para las posteriores políticas sociales (9 p.99-101).

Las señoras de ALPI se reunieron, en principio, desde la sensibilidad basada en sentimientos religiosos hacia una situación de enfermedad que afectaba, en algunos casos, directamente el seno de sus propias familias. Hacia la década de 1940 aún resultaba incierta la posibilidad de controlar el contagio de la enfermedad y se hacía necesario implementar los onerosos y prolongados tratamientos de rehabilitación entre las familias más pobres.

No obstante, con el transcurso del tiempo su compromiso y especialización en temas de discapacidad y rehabilitación se vieron afianzados más allá de las problemáticas relacionadas con la poliomielitis. Hacia 1957, las autoridades sanitarias, preocupadas por los altos costos de adquirir una vacuna importada, convocaron a ALPI para la realización de la Primera Campaña de Recaudación Pro Vacunación, con el propósito de reunir fondos entre la población y de ese modo morigerar el impacto económico que la inmunización a gran escala significaría para las arcas estatales. A mediados de 1959, ALPI reconoció en la vacunación una de las actividades más relevantes en su impacto de acción social. Con la colaboración de un gran número de voluntarios, la entidad vacunaba gratuitamente a todos aqueIlos que no podían pagar la vacuna. Por otra parte, el impacto de la aplicación masiva de la vacuna, como una herramienta de comprobada eficacia para el control del contagio de la poliomielitis, puso en jaque los fines explícitos que sostenían a ALPI. Así, en 1961, la institución se vio obligada a rediseñar su misión y sus objetivos para continuar en vigencia luego de 20 años de "obra" y de haber logrado fuertes vinculaciones civiles y estatales en los cambiantes escenarios que se presentaron durante ese lapso. Esto representó para ALPI no solo la redefinición de sus fines y el desafío de adaptación de su capacidad técnica y administrativa acorde con nuevas estructuras y perfiles profesionales ajustados a otras problemáticas y tipo de discapacidades, sino que significaría también un esfuerzo necesario para sobrevivir sin secuelas a la poliomielitis.

Así pues, a través de una alianza médico-social ALPI conformó un modelo de asistencia que combinaría la intervención pública, la filantropía basada en sentimientos religiosos y la ciencia, por cierto a tono con una larga continuidad histórica que asociaba al Estado y a los organismos privados en la distribución de las responsabilidades del cuidado de los niños, pobres y enfermos (9).

Esta institución realizaba múltiples actividades en diferentes ámbitos e instancias. Las primeras acciones de ALPI se tradujeron en 
prestaciones de ayuda a los enfermos que se asistían en los hospitales de la ciudad de Buenos Aires, a través de la provisión de aparatos ortopédicos, dinero para traslados, ropas y medicamentos. En síntesis, la actualización científica, el desarrollo y la difusión de la metodología de abordaje, y la accesibilidad a los aparatos de ortopedia fueron tres de los tópicos en los que concentraron los recursos. Las estrategias de recaudación de fondos estaban basadas en las colectas populares, las donaciones y legados, y los eventos sociales y culturales a beneficio. En este sentido, la metodología utilizada por la Fundación Nacional contra la Poliomielitis en EE.UU., que solventaba gran parte de las iniciativas en materia de tratamientos de rehabilitación e investigaciones, constituyó un ejemplo a seguir $(10,11)$. En publicaciones de prensa, en las memorias institucionales y en folletería institucional se difundían los nombres y hasta las fotografías de los patrocinadores que realizaban donaciones importantes en una demostración de la concepción de filantropía, no solo como gesto de utilidad y acción social sino también como expresión de prestigio social.

\section{MEMORIAS DE UN INICIO}

Ahora bien, en esta reseña tomaremos como punto de partida las memorias institucionales de ALPI (Figura 1 y Figura 2) -especialmente la de su primer año de existencia- para analizar algunos aspectos de su fundación prestando atención a dos de las personas que constituyeron el binomio médico-social que condujo el accionar de la entidad desde 1943 hasta 1950: la asistente social Marta Ezcurra y el cirujano ortopédico Marcelo Fitte.

Analizar dichos documentos se convierte, además, en una oportunidad para reconstruir algunas de las actitudes y respuestas concretas de la ciudadanía ante la conmoción de las epidemias de polio y contribuir a pensar la relación entre los recursos movilizados por la sociedad civil y la conformación del problema de la rehabilitación como una cuestión de interés público, digna de ser tenida en cuenta por los centros de poder. La hipótesis que sostenemos es que para desarrollar el interés fundamental de practicar una modalidad de tratamiento que aún requería de comprobación, galenos y señoras constituyeron una alianza médico-social sostenida en un andamiaje de ideas científicas y cristianas que legitimaron sus intervenciones en la vida pública y en el cuerpo de los enfermos, objeto de sus desvelos y sus prácticas.

\section{Marta Ezcurra: ¿huellas del catolicismo social?}

Las primeras reuniones que dieron impulso a la organización de esta Asociación se realizaron durante el año 1943 en la residencia de los esposos Juan Bernardo Sullivan y Juana Berthet, ubicada en un acomodado barrio porteño. Allí sentaron las bases de la entidad y tuvo lugar la primera reunión de los socios fundadores.

El matrimonio Sullivan, que "sentía el sufrimiento de la enfermedad en carne propia" (8 p.3), brindó apoyo económico no solo con importantes y sostenidas donaciones particulares sino también a través del aporte monetario de la Forestal Argentina SA, compañía presidida por aquellos años por Juan Bernardo Sullivan (12). El matrimonio se hizo cargo del costo de los primeros meses de alquiler de la primera sede de la Asociación, en la Av. Paseo Colón 221, $2^{\circ}$ piso, hasta que fuera luego cedido el uso de ese mismo espacio por la Forestal Argentina S.A. hasta 1962, año que se trasladarían a una sede administrativa propia (b).

Marta Ezcurra ocupó los cargos de presidenta y vicepresidenta alternadamente durante los primeros seis ejercicios (1943-1950) y permaneció vinculada a esta entidad a lo largo del período analizado. Esta mujer traía consigo un bagaje de recursos profesionales y vinculaciones políticas que, sumados a los de las damas que conformaron la Comisión Directiva (Figura 1) -muchas de ellas de apellidos notables- y a las donaciones de las familias Fitte y Sullivan, establecieron las bases del capital inicial de la Asociación.

Al asumir el cargo, Marta contaba con una sólida y activa experiencia en el movimiento de Acción Católica, organización eclesial no clerical dedicada a la evangelización y la promoción de los valores cristianos. Sus inicios en el servicio social habían comenzado en 1915 cuando, recién 
Figura 1. $1^{\circ}$ Comisión Directiva y Comisión Honoraria Consultiva de Médicos de la Asociación para la Lucha contra la Parálisis Infantil, 1944.

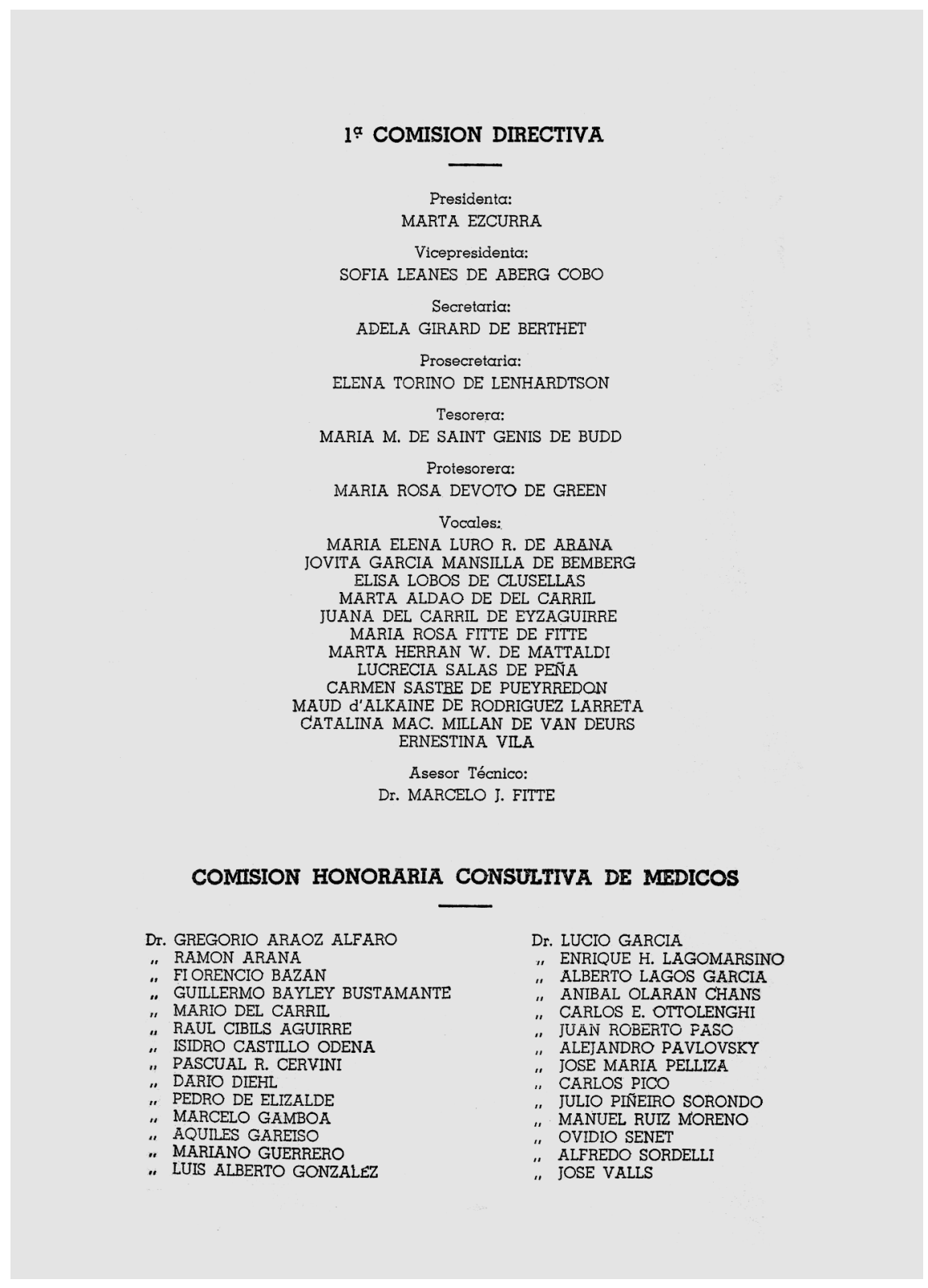

Fuente: Asociación para la Lucha contra la Parálisis Infantil (8).

egresada del Colegio Sagrado Corazón de Almagro, ingresó a la Congregación Hijas de María. Participó de reuniones de estudio y formación apostólico-social en la congregación Hermanas de la Asunción. En 1925, comenzó a trabajar en El Centavo, una asociación destinada a asistir a mujeres necesitadas a través de la provisión directa de alimentos y de talleres de costu- ra como medio de ganarse el sustento y de cuyo Consejo Superior formaría parte luego.

En el año 1930 había ingresado a la recientemente fundada Escuela de Servicio Social del Museo Social Argentino, primera del país y de latinoamérica, donde obtuvo el título de asistente social, en 1933. En 1931 fue nombrada presidenta del Primer Congreso Superior de las Jóvenes de la 
Figura 2. Memoria de la Primera Asamblea General Ordinaria de la Asociación para la Lucha contra la Parálisis Infantil, 1944.

\section{MEMORIA}

La Comisión Directiva, en cumplimiento de lo dispuesto por el ortículo $15^{\circ}$ de los Estatutos Sociales, ha convocado a esta Primera Ascmblea General Ordinaria, para dar cuenta de la constitución definitiva de la Asociación, el día 17 de Diciembre de 1943, de la labor desarrollada hasta el 30 de Junio de 1944 y de la marcha de sus gestiones.

Como es sabido, la Asociación nació del dolor cristianamente sobrellevado. Dos jóvenes señoras que sentían los sufrimientos de la enfermedad en carne propic, porque lo sentían en sus hijos, decidieron ocuparse de las formilias pobres en las mismas dolorosas circunstancias.

Queda, pues, constancia de los nombres de las señoras de Berthet $\mathrm{y}$ Lenhardtson $\mathrm{y}$ de dos amigas que apoyaron esta obra $\mathrm{y}$ juntas comenzaron la campaña: son ellas las señoras de Budd y Mattaldi.

Constituída, como decía, el 17 de Diciembre, esta Comisión Directiva designó por aclamación al doctor Marcelo J. Fitte como Asesor Técnico, dedicando sus primeras gestiones a obtener la inscripción en el Registro Nacional de Asistencia Social, donde quedó registrado bajo el Ni 3.244 .

Estudió luego un proyecto de Estatutos y una vez llenadas todas las formalidades ante la. Inspección General de Justicia, el Poder Ejecutivo de la Nación, concedió por decreto $\mathrm{N}^{\circ} 9.282 / 44$ de fecha 13 de Abril de 1944 la Personalidad Jurídica. Vaya nuestro agradecimiento al doctor Benjamín García Victorica por la redacción de los Estatutos y al doctor Guillermo Peña por tramitar ante el Ministerio del Interior.

A partir de aquella fecha, la Asociación se encuentra legalmente capacitada para desenvolver sus actividades. 
Figura 2. Continuación.

Una medida de singular importancia fué la de invitar a los señores Médicos cuya actuación profesional se halla relacionada con el problema de la Parálisis Infantil a cooperar en nuestra obra y nos satisface destacar que respondieron con toda solicitud a nuestro requerimiento. Quedó así constituída la Comisión Honoraria Consultiva de Médicos, cuya nómina inicial se hallará al comienzo de esta Memoria.

Posteriormente se ofreció a todos ellos el apoyo para sus enfermos de Parálisis, cuyas condiciones económicas así lo justificaran.

Dentro de los recursos iniciales de la Asociación, la Comisión Directiva ha iniciado ya la prestación de su ayuda a tales enfermos, algunos de los cuales se asisten en establecimientos hospitalarios de esta Capital, proveyendo los aparatos ortopédicos prescriptos.

Se cuenta con los servicios de una Visitadora de Higiene, cuya misión es de verificar en los domicilios de los enfermos, las condiciones en que se llevan a efecto los prescripciones médicas y las posibilidades económicas de los familiares. La Sub-comisión de Assistencia Social compuesta por las señoras Juana del Carril de Eyzaguirre, Lucrecia Salas de Peña, Adela Girard de Berthet, María Magdalena de Saint Genis de Budd, Marta Herrón Witcomb de Mattaldi, María Rosa Fitte de Fitte y María Luisa Noetinger de Peluffo Alemón complementa personalmente las visitas domiciliarias para realizar además acción educativa y moral. En virtud de estas visitas, la Sub-comisión de Señoras aconsejó la donación de aparatos ortopédicos para 10 niños, llenándose las órdenes por un valor de $\$ 2.000 \mathrm{~m} / \mathrm{n}$.

\section{3}

Como resultara indispensable encarar un plan de difusión y propaganda para dar a conocer al público la constitución de la Asociación y organizar una colecta que permitiera reunir los fondos necesarios para la ejecución de sus fines, la Comisión Directiva designó dos Sub-comisiones especiales: la de Prensa y Propaganda y la de Colecta.

La Sub-comisión de Prensa y Propaganda, integrada por las señoras Lucrecia Salas de Peña, María Rosa Fitte de Fitte, Adela Girord de Berthet, y los señores Alberto R. Olguín y Enrique Diosdado, presentó un amplio plan de publicidad y recaudación de fondos. Dado el momento nacional se aprueba solamente un plan mínimo, el cual comprende: 
Figura 2. Continuación.

a) La organización de un Concurso de "affiches" con el lema "Levántate y Anda", sugestiva frase evangélica.

b) Una campaña financiera, a realizarse en la primera quincena de Septiembre próximo.

Para efectuar esta campaño se constituyó la Sub-comisión de Colecta bajo la Presidencia de la señora Juana del Carril de Eyzaguirre ayudada por las señoras Elena Torino de Lenhardtson, María Rosa Devoto de Green, Morta Herrán Witcomb de Mattaldi, María Rosa Fitte de Fitte, Elisa Lobos de Clusellas, Lucrecia Salas de Peña, María Magdalena de Saint Genis de Budd, Catalina Marc. Millan de Van Deurs, Carmen Sastre de Pueyrredón, Adela Girard de Berthet, Jovita García Mansilla de Bemberg y Maud d'Allkaine de Rodríguez Larreta.

Los "affiches" han llegado en número de 74 y serán exhibidos en la casa Gath \& Chaves Ltda., en un salón cedido gentilmente al eíecto.

\section{3}

La Comisión Directiva ha encarado también el problema relativo a las reeducaciones y actualmente tiene en estudio un proyecto para contribuir a la preparación de este personal en la Capital y en el Interior, propendiendo orgónicamente a la obra de reeducación funcional de los enfermos.

Consistirá en costear 10 becas para que enfermeras de las provincias puedan beneficiarse con los cursos especializados que el doctor Marcelo J. Fitte ha de dictar en el Hospital de Niños.

Coincidentemente con dicho propósito se ha creado la Bolsa de Trabajo, en la cual se inscriben los enfermos cuya aptitud permite emplearlos en distintas ocupaciones accesibles a sus medios. Ia Asociación procura proveerlos de trabajos adecuados, auspiciándose además la obra que realiza la Sociedad "Amigos de la Escuela ambulante" y que consiste en la enseñanza a domicilio de los niños inválidos.

\section{$3 \frac{2}{1}$}

La Comisión Directiva se hace un deber en dejar constancia del apoyo unánime que ha recibido de todos los colaboradores que de un modo $u$ otro han prestado su eficaz y desinteresado 
Figura 2. Continuación.

concurso para lograr la constítución, organización y funcionamiento de la Asociación, debiendo mencionar: las facilidades que fueron otorgadas por la Dirección de Salud Pública y por el Registro Nacional de Asistencia Social; el apoyo de la prensa de todo el país, por el generoso espacio que dedicara a nuestra Asociación; la Sociedad de Beneficencia de la Capital, por los cursos de especialización para enfermeras, en su Servicio de Parálisis Infantil del Hospital de Niños; el señor Max Von Buch, por su proyecto de organizar una Exposición de Arte Gótico y Renacimiento a fin de año.

Debe asimismo esta Comisión Directiva destacar con gratitud el decidido apoyo que prestaron el señor Juan Bernardo Sullivan y Señora desde el momento mismo de gestarse la idea de la creación de esta entidad. Facilitaron gentilmente su residencia en la A.v. Alvear 3762 donde se sentaron las bases de la entidad y tuvo lugar la primera reunión de los Socios Fundadores; y costean, además, el alquiler actual de la sede.

La Comisión Directiva, confía en que dado el resultado deI primer llamado para formar esta Āsociación, inspirada en propósitos de bien común, y la franca acogida que ha recibido de núcleos calificados de personas, de las autoridades oficiales y del público en general, podrá afianzarse en lo futuro, para cumplir dentro de sus limitados medios humanos, pero con verdadero fervor, la inspiración de la frase evangélica: "Levántate y Anda". 
Acción Católica por el arzobispo Santiago Copello. Al año siguiente, realizó un viaje a Europa, donde mantuvo una audiencia privada con el Papa Pío XI en representación de la flamante Asociación de Jóvenes de la Acción Católica. En este viaje se vinculó con representantes de la Cruz Roja Internacional y tomó contacto con programas de asistencia social europeos que adherían a la idea de que ofrecer trabajo era mejor que ofrecer limosna, a la vez que dignificaba la condición humana, en sintonía con la propuesta de monseñor Miguel de Andrea, que alzaba su voz para denunciar la explotación de las obreras. También trabajó activamente en la promoción de la coordinación de la obras privadas que se autodefinían como obras neutras y confesionales. En 1952 participaría de la Organización de las Naciones Unidas cumpliendo funciones en países de América Latina y en 1955 fue nombrada por el gobierno de facto como Directora de Asistencia Integral del Ministerio de Asistencia Social y Salud Pública (13-15).

Cuando se relacionó con ALPI, Ezcurra había viajado a EE.UU. junto con otros dirigentes católicos latinoamericanos y había visitado los sindicatos del vestido de Chicago, Nueva York, los tribunales de trabajo y las comisiones del salario. Además, era directora de la Escuela de Servicio Social del Instituto de Cultura Religiosa Superior fundada por el cardenal Luis Copello y dictaba cursos de Doctrina Social Católica. También dirigía la filial argentina de la Unión Católica Internacional de Servicio Social, una de las entidades más represetantivas del catolicismo conservador. Indudablemete, había logrado conquistar un importante lugar en el terreno del catolicismo social debido a una activa participación en múltiples espacios.

Recordemos que, según describe Omar Acha, desde la década de 1930 estaban en escena no solo las mujeres católicas de elite, sino que existían otros grupos de mujeres que incluían entre sus filas a los sectores medios y se daban diferentes estrategias de actuación desde una cosmovisión protectora de los ideales de la familia católica (16 p.199). Además, las características fragmentarias del proceso de centralización de las políticas asistenciales iniciado en Argentina desde la década de 1940 permitió la existencia de otras variadas organizaciones civiles -laicas o religiosas- que atendían diferentes situaciones sanitarias y sociales (17). Entre ellas, ALPI, quien lejos de suscitar el interés estatizador, desarrolló acciones concurrentes y complementarias a las políticas sanitarias del Estado -especialmente durante las acciones de inmunización- en una especie de gestión asociada que por un lado favorecía la expansión y el fortalecimiento de la entidad civil que ganaba autonomía y prestigio en sus áreas de influencia y, por el otro, alivianaba el esfuerzo del Estado para atender el problema de los brotes epidémicos y el tratamiento de las personas afectadas por la enfermedad (18).

En este contexto entonces, se puede entender como rasgo natural de la época que las mujeres integrantes de ALPI, ejemplo de la figura católica femenina por excelencia, representada tradicionalmente por mujeres de buena posición económica, confiaran la guía y el asesoramiento para dar los primeros pasos de la entidad a una destacada referente del pensamiento femenino del catolicismo social, a la vez que convocaban la participación de otras mujeres de sectores económicos medios.

Ezcurra había recorrido temas como lo económico-social, el apostolado, la familia, el Estado, la justicia, la caridad, la Iglesia y la acción social, en un lenguaje dirigido a la comprensión del público en general. Sus enseñanzas y consejos reflejaban el contexto de crisis de la legitimidad liberal, y eran obedientes al discurso de la jerarquía eclesiástica que pretendía imponer una visión del mundo en todos los ámbitos de la vida, tanto públicos como privados. La trayectoria profesional de Marta Ezcurra y su anclaje político y social dan muestra de ello, y explicarían su presunto y potencial interés por apoyar el surgimiento de esta entidad como posibilidad de extender su influencia en la sociedad civil, en un marco que permitía diferentes matices y estrategias en las formas de gestionar la asistencia social.

\section{Marcelo Fitte y el rol de las mujeres para una "medicina elástica y social" (c)}

El papel del cirujano ortopédico Marcelo Fitte fue influyente en la historia de los primeros años de la Asociación y también en las ideas que, 
respecto del tratamiento de la poliomielitis, primaron en ese período. Fitte encontró en ALPI las condiciones para el desarrollo de su terapéutica, un contexto propicio que quizá no era óptimo en el Hospital de Niños donde también se desempeñaba como jefe de la Sala de Poliomielitis. El Dr. Marcelo Fitte, reconocido como mentor y creador de ALPI, tenía una opinión asumida con respecto al rol de las mujeres como mediadoras entre el mundo de los enfermos y el mundo médico a través de las visitas domiciliarias y la reeducación, entendiendo por ello la trasmisión y supervisión por parte de las reeducadoras de determinados cuidados específicos que era necesario aplicar a los enfermos en las distintas etapas del tratamiento y relevando, a su vez, las necesidades de las familias que podían significar un obstáculo para la terapéutica. En 1938, en su propuesta de organización para un servicio de poliomielitis, reconocía la importancia de las condiciones físicas y espirituales femeninas para el éxito de un proyecto sanitario moderno, a la altura de otros similares en el mundo.

En este orden de cosas, es a veces extraordinaria la sugestión que un físico agradable y buenas maneras pueden ejercer. Tal vez por ello, es que las mujeres han mostrado poseer mejores cualidades que los hombres y esa función de reeducación está, en casi todo el mundo, encomendada a manos femeninas. (19 p.140)

En 1929, Fitte fue designado como médico por la Sociedad de Beneficencia en el Servicio de Cirugía del Hospital de Niños. En 1939 creó el Servicio de Parálisis Infantil (Pabellón Tamini) de ese hospital. Un año antes había obtenido el cargo de Profesor Adjunto de la Cátedra de Ortopedia de la Facultad de Ciencias Médicas de la Universidad de Buenos Aires, donde había comenzado como adscripto en 1924 (20).

No obstante sus antecedentes profesionales y docentes, las ideas sobre el tratamiento ortopédico precoz que Fitte venía intentando aplicar en el pabellón Tamini del Hospital de Niños no siempre fueron vistas con simpatía por algunos de sus colegas, que preferían la aplicación de técnicas de electroestimulación y de movilización kinésica luego de superada la etapa aguda y sub aguda de la enfermedad, en oposición a la temprana intervención que proponía el ortopedista.

Hacia 1942, Fitte había Ilevado maestras para que dieran clases en la sala de niños internados con secuelas deformantes que permanecían allí durante años (19).

El cirujano sostenía que el tratamiento ortopédico precoz era el recurso más eficaz y adecuado para tratar la enfermedad, y que la función indiscutida de liderazgo debía recaer en el médico cirujano ortopedista. Dicho tratamiento se basaba en la intervención temprana del ortopedista desde la fase aguda, la utilización de aparatos ortopédicos, las cirugías correctivas, la reeducación muscular y los masajes (en mesa o en el agua), la constante vigilancia de las posiciones adecuadas del cuerpo y el seguimiento de la evolución del paciente, que se realizaría en el hogar. Proponía técnicas especiales de manipulación y corrección de los cuerpos afectados para evitar y prevenir las deformidades $(19,21,22)$.

Fitte destacaba la necesidad de crear verdaderos servicios elásticos, capaces de responder a las epidemias desiguales. Dicha elasticidad estaría dada por la capacidad de aumentar rápidamente el número de camas en el hospital y por el seguimiento de los pequeños pacientes en sus respectivos domicilios, que sería realizado por las visitadoras.

\footnotetext{
...recurriendo a la ayuda de las visitadoras, quienes estarán encargadas de comprobar si los pacientes son mantenidos en las posiciones adecuadas, si sus aparatos siguen desempeñando el rol para el cual se hicieron y si por cualquier razón es necesario que concurran al servicio hospitalario [...] creando así en esta forma un verdadero servicio externo, que seguirá al niño como si estuviera internado, sin necesidad de cargar con el costo de la internación. (21 p.2347)
}

La medicina debe tener alcance social y por ello nos atrevemos a recordar, que no basta curar o mejorar estos enfermos, debiendo llevar la acción más allá. Esta obra social no es en nuestro país atendida con la intensidad debida, por eso subrayamos aquí esta deficiencia. (22 p.92)

Queda reflejado en sus palabras que no solo el deseado carácter de elasticidad era un rasgo que 
se materializaría a través de las visitas al hogar; también el pretendido alcance social de la medicina, aquel que iba más allá de las acciones de curar o mejorar a los enfermos, descansaría en estas mujeres que atravesaban las puertas del hogar y del hospital como mediadoras entre los asistidos y la institución sanitaria a la que ellas representaban. Efectivamente, Fitte no solo pensaba en un tratamiento médico sino que proponía una organización asistencial y social que lo sustentara, y es en ese aspecto donde toma importancia la asociación con el grupo de mujeres de ALPI.

Cinco años después de que Fitte hubiera comenzado a aplicar su programa en el Hospital de Niños, ALPI comenzó sus funciones en concordancia con los lineamientos médicos del cirujano ortopedista. En esa oportunidad fueron las señoras de la Subcomisión de Asistencia Social, basadas en cierta autopercepción que las definía como las más adecuadas para garantizar la acción educativa y moral en el ámbito privado de los hogares, las que asumieron la realización de las visitas domiciliarias.

No obstante que el hecho de adoptar la reeducación domiciliaria como práctica institucionalizada significaba su gradual reconocimiento como una práctica sanitaria que requería dominar conocimientos y técnicas precisas para el manejo y control de los cuerpos de los enfermos -lo cual justificaba su paulatina profesionalización- no por ello abandonaría los términos morales y educativos que le habían dado origen.

Dichos términos morales estaban basados en la idea del niño inválido como objeto de la caridad y de la asistencia: el niño de familias de escasos recursos sería tutelado en mayor medida, estudiado y rehabilitado en todos los casos en que fuera posible.

Ciertamente, la práctica de la reeducación domiciliaria cumplía un papel clave en el proyecto sanitario de Fitte y de ALPI: debía ser eficaz no solo como intervención sanitaria y educativa que asegurara el cumplimiento de las indicaciones médicas, sino que su sesgo moral quizá se engrandecía calladamente al legitimar desde la práctica un modelo de atención sanitaria que ubicaba una parte del peso de la deficiencia dentro de los límites de la unidad familiar y la responsabilidad individual.

\section{¡Levántate y anda!: ¿hacia un modelo de rehabilitación?}

La sugestiva frase evangélica fue pronunciada en las Memorias de ALPI del primer ejercicio para referirse al desafío de afianzarse en el futuro para "cumplir dentro de sus limitados medios humanos, pero con verdadero fervor, la inspiración..." que tal frase plantea (8 p.8). También fue el lema de un concurso de afiches realizado en 1943, del que surgió el distintivo de la Asociación, seleccionado entre 74 trabajos que fueron exhibidos en la Casa Gath y Chaves Ltda., en un salón cedido a tal efecto.

Desde la perspectiva católica, la función maternal es la que acepta y justifica todo sacrificio y entera entrega. Es a esa idea de madre capaz de soportar todo infortunio, y a través de ella a la sociedad toda, a la que se le dice: ¡levántate y anda! Nadie mejor que otra madre para animar y propender a la superación de la parálisis y la impotencia, provocadas no solo por el virus sino por el temor al contagio.

El contenido moral de las intervenciones destacaba las cualidades y la voluntad individual del enfermo que, conjugados con la responsabilidad y dedicación que la familia pusiera en juego en cada caso, rendiría sus frutos en los resultados obtenidos en la rehabilitación.

Los premios ALPI al esfuerzo y el tesón constituyen una muestra de ello. Los valores distinguidos eran, justamente, el esfuerzo por superar individualmente la dificultad, la proyección social lograda a partir de dicha superación y la contribución solidaria realizada en su comunidad. Los premios se entregaban en emotivas ceremonias en las cuales los elegidos recibían el reconocimiento público por su esfuerzo a la vez que expresaban su gratitud, y destacaban la abnegación y la entrega femenina de las representantes de ALPI.

Los seleccionados eran personas consideradas como recuperadas y útiles a la sociedad, que habían alcanzado títulos terciarios o universitarios, o que se habían destacado en el deporte o en ámbitos artísticos, que demostraban a través de sus trayectorias personales, y de acuerdo con los valores de la época, la posible eficiencia y alcances de la rehabilitación (d).

La condición femenina y la maternidad, sumadas al ejercicio del poder que naturalmente 
se les adjudicaba a las mujeres para ejercer el control sobre niños y enfermos constituyeron la base que autorizaba a estas mujeres para asumir tales responsabilidades. La mirada de clase, el lugar generizado de las funciones arrogadas y sus vinculaciones familiares y personales formaron parte de los recursos simbólicos y materiales que se pusieron en juego para el despliegue de las actividades de esta institución. Muestra de ello son los innumerables eventos sociales y culturales que organizaban para recaudar fondos pero que, al mismo tiempo, aumentaban sus ámbitos de sociabilidad, cercanía de clase y pretendida distinción social. Los té-canasta, los sofisticados almuerzos étnicos, los cócteles, los distinguidos desfiles de modas, los remates de obras de arte se realizaban en los domicilios particulares o en selectos establecimientos capitalinos. La demostración de riqueza, lujo y distinción social estaba legitimada por los valores fundados en la caridad cristiana y el bien común.

En la búsqueda de recursos humanos necesarios para llevar adelante su lucha recurrieron al reclutamiento de otras mujeres de clase media y de sectores populares que participaban como voluntarias en distintas funciones de cuidado y asistencia directa (24-26) (e).

La administración y la toma de decisiones estaban en manos de la comisión directiva, exclusivamente femenina. Los diferentes espacios de participación que ofrecían a otras mujeres presentaban una doble vinculación. En primer lugar, a ellas mismas como representantes de la clase dominante. En segundo lugar, a la medicina en tanto representante de una disciplina profesional que concentraba altos grados de autonomía y un saber reconocido como científico y legítimo, que mejoraría las naturales condiciones femeninas para el cuidado a través de las capacitaciones en técnicas rehabilitadoras (masajes, fomentos, movilizaciones). La gradual profesionalización de estas funciones de cuidado -al igual que en el caso de las asistentes sociales y las enfermeras- dio la oportunidad a las mujeres de acceder al mundo del trabajo y de la educación desde su costado natural femenino y subordinado (26 p.120).

Es necesario mencionar que algunas de las mujeres que participaron en las primeras comisiones directivas de ALPI y se capacitaron en las técnicas especializadas para tratar las secuelas de la enfermedad (masajes, fomentaciones ( $f$ ), movilizaciones y reeducación muscular) fueron las esposas e hijas de médicos que se habían especializado en tratar la poliomielitis. A pesar de que ellas ocuparon los cargos jerárquicos que se organizaron en torno a esas actividades, mantuvieron un vínculo de subordinación determinado por la valoración de dichas tareas, consideradas de menor rango profesional y académico. La elección de ejercer una ocupación en función de acompañar el desarrollo profesional del marido o el padre reflejaba los valores de la época, que otorgaban a las mujeres un lugar secundario con respecto a ellos (24 p.34). Además, estos primeros cursos de capacitación bosquejaron la demanda y los antecedentes para la posterior creación de centros de formación de algunas profesiones consideradas colaboradoras de la medicina.

\section{PALABRAS FINALES}

ALPI reconoció en las ideas de organización y tratamiento del ortopedista y sus seguidores una necesaria fuente de legitimidad científico-técnica que la asociación por sí sola no podía garantizar. Por su parte, el grupo de médicos reconoció en ALPI la oportunidad de poner un pie en el ámbito privado para afianzar y desarrollar una modalidad de tratamiento que se reprodujo en forma paralela y complementaria con el sector estatal, a la vez que la ortopedia como especialidad médica generó un espacio de disputa por la hegemonía en el terreno de las enfermedades invalidantes.

A lo largo de su trayectoria, ALPI logró conquistar legitimidad científica en el campo de las discapacidades motrices y demostró capacidad suficiente para movilizar recursos no solo en la ciudad de Buenos Aires y el conurbano, sino también en otros centros del interior del país, tales como Tucumán, Córdoba, Santa Fe, Jujuy y localidades de la provincia de Buenos Aires. Esta asociación ofreció viabilidad institucional para aplicar novedosas y variadas técnicas aprendidas en el extranjero, y fue precursora de la aparición de nuevas profesiones y especialidades. Tal es el caso de las carreras de terapia ocupacional, terapia física y las tecnicaturas en ortesis y prótesis. 
De ese modo contribuyó, principalmente, en el alivio de las tensiones inherentes a la problemática social que la niñez inválida representaba, proveyendo herramientas cuya eficacia ya había sido comprobada, que fueron tenidas en cuenta para la posterior puesta en práctica de las políticas públicas instrumentadas a partir de 1956 (27).

En efecto, la actuación de ALPI ofrece algunos indicios que revelan cómo se tradujeron en los hechos las ideas de carácter filantrópico con las de carácter médico-científico, las ideas de caridad católica versus la solidaridad laica. En esa ambigua trama se entremezclaban concepciones científicas y preceptos de caridad y filantropía con ciertas ideas de reconocimiento de los derechos de las personas con discapacidad (especialmente referidos al derecho a la educación y al trabajo) por parte de las autoridades oficiales (2830). En ese sentido, tal como argumenta Perelmiter con respecto a las asistentes sociales, este caso refleja un "proceso de sincretismo entre esferas de valor" que amalgaman ciencia, religión y Estado (15 p.43); una alianza médicosocial basada en un encuentro de racionalidades diferentes que dieron tonalidades de grises en un contexto de incertidumbres y de formas particulares de articularse que exigían nuevas y urgentes respuestas para enfrentar el problema de los niños inválidos.

Las estrategias de asistencia médico social de ALPI implicaban, al menos en la etapa inicial que aquí se analiza, las tareas de asistir, proteger e incluir socialmente a los niños afectados con secuelas de poliomielitis. Algunas de sus características fueron peculiares y distintivas, la presencia institucional lograda entre la gente común a través de las campañas de recaudación con las alcancías y los elegantes eventos, los premios ALPI, la capacidad de extender su influencia a las provincias del interior y de participar en variados espacios científicos y sociales de otros países formaron parte de dichas estrategias. Algunos de estos rasgos presentaron matices en los modos de ejercer la filantropía que bosquejaron, a través de la alianza médico-social y de las gestiones asociadas al Estado, una forma de accionar que sentó precedentes y viabilidad para la posterior puesta en práctica de un modelo de rehabilitación que se afianzaría en nuestro país a partir de la década de 1960

\section{NOTAS FINALES}

a. Esta expresión fue tomada del discurso del Dr. Félix Liceaga (5) en la defensa del proyecto de Ley sobre creaciones, en la Capital Federal, de un hospital de niños para enfermedades infecciosas y de un instituto de Heine Medin, presentados en la Cámara de Diputados el 21 de agosto de 1946.

b. La Forestal fue una empresa argentina de origen inglés que se instaló en nuestro país en 1872 a partir de un empréstito con la compañía Murrieta de Londres. Explotó 1.500.000 de hectáreas de quebrachales del Chaco para exportar tanino, postes y durmientes para el ferrocarril. Se retiró del país en 1966 debido a la caída de los aranceles internacionales de la madera y el tanino. En su paso de casi 90 años dejó nefastas consecuencias económicas, ecológicas y sociales. Agotó el recurso forestal, pero ganó un lugar destacado en nuestra historia de explotación, represión, muerte y corrupción. Ver Virasoro (12). c. Así es como caracterizaba Marcelo Fitte a los servicios que deberían ocuparse de la poliomielitis (20).

d. ALPI mantiene en vigencia estos premios, a través del Concurso Bienal Nacional de ALPI. Los aspirantes son presentados por entidades o personas individuales que conocen los méritos personales y sociales de cada uno.

e. La idea de ampliación de las bases sociales del sistema de beneficencia es de Eduardo Ciafardo (25).

f. Las fomentaciones consistían en aplicar telas calientes sobre los miembros paralizados. Para ello se utilizaban telas especiales que se calentaban con agua caliente y luego se escurrían en un aparato con rodillos. Se indicaban en la etapa aguda de la enfermedad y se complementaban con la movilización pasiva de los miembros afectados. Su objetivo era reducir la espasticidad y su consecuente deformación articular y muscular. 


\section{REFERENCIAS BIBLIOGRÁFICAS}

1. Invaldi A. Epidemiología mundial de la poliomielitis. Revista de la Asociación Médica Argentina. 1956;70(827-830):265-269. [Material de Archivo]. Localizado en: Buenos Aires: Biblioteca Central Juan José Montes de Oca, Facultad de Medicina, Universidad de Buenos Aires.

2. Bottinelli P. Estudio estadístico de la poliomielitis. La Semana Médica. 1953;58(33):295-302. [Material de Archivo]. Localizado en: Buenos Aires: Biblioteca Central Juan José Montes de Oca, Facultad de Medicina, Universidad de Buenos Aires.

3. Vilches A. Etiología, patogenia y epidemiología de la poliomielitis. Archivos Argentinos de Pediatría. 1956;45(3):175-192. [Material de Archivo]. Localizado en: Buenos Aires: Biblioteca Central Juan José Montes de Oca, Facultad de Medicina, Universidad de Buenos Aires.

4. Garrahan J. Medicina infantil: Pediatría y Puericultura. 7a ed. Buenos Aires: El Ateneo; 1951. [Material de Archivo]. Localizado en: Buenos Aires: Biblioteca Central Juan José Montes de Oca, Facultad de Medicina, Universidad de Buenos Aires.

5. Liceaga F. Proyecto de Ley sobre creaciones, en la Capital Federal, de un hospital de niños para enfermedades infecciosas y de un instituto de Heine Medin. Buenos Aires: Secretaría de Presidencia; 21 ago 1946. [Material de Archivo]. Localizado en: Buenos Aires: Hemeroteca del Congreso de la Nación.

6. Buzzi A, Rosenwurcel H. Historia de la Poliomielitis. Revista Asociación Médica Argentina. 1967;81(8):393-395. [Material de Archivo]. Localizado en: Buenos Aires: Biblioteca Central Juan José Montes de Oca, Facultad de Medicina, Universidad de Buenos Aires.

7. Ministerio de Bienestar Social, Subsecretaría de Salud Pública, Departamento de Atención Médica. Historia de la epidemiología de la poliomielitis en la República Argentina. Boletín Epidemiológico Nacional. 1977;8(5). [Material de Archivo]. Localizado en: Buenos Aires: Biblioteca Central Juan José Montes de Oca, Facultad de Medicina, Universidad de Buenos Aires.

8. Asociación para la Lucha contra la Parálisis Infantil. Memorias y Balance: Primer ejercicio. Buenos Aires: ALPI; 1944. [Material de archivo]. Localizada en: Buenos Aires: Instituto Marcelo Fitte.
9. Guy D. Las mujeres y la construcción del Estado de Bienestar: Caridad y creación de derechos en Argentina. Buenos Aires: Prometeo; 2011.

10. Oshinsky D. Polio: An American story - The crusade that mobilized the nation against the 20th century's most feared disease. Nueva York: Oxford University Press; 2005.

11. Berg R. La lucha contra la poliomielitis. Buenos Aires: Nova; 1959.

12. Virasoro R. La Forestal Argentina. Buenos Aires: Centro Editor de América Latina; 1971.

13. Alayón N. Historia del trabajo social en la Argentina. Buenos Aires: Espacio Editorial; 2007.

14. Grassi E. La mujer y la profesión de Asistente Social: El control de la vida cotidiana. Buenos Aires: Humanitas; 1989.

15. Perelmiter L. Micropolítica burocrática y asistencia social: Agentes y prácticas en el Ministerio de Desarrollo Social de la Nación, Argentina (2003-2008). [Tesis de doctorado]. Buenos Aires: Facultad de Ciencia Sociales, Universidad de Buenos Aires; 2011.

16. Acha O. Catolicismo social y feminidad en la década de 1930: de damas a mujeres. En: Acha O, Halperín $\mathrm{P}$, editores. Cuerpos, géneros, identidades: Estudios de historia de género en Argentina. Buenos Aires: Ediciones del Signo; 2000.

17. Ramacciotti K. La política sanitaria del peronismo. Buenos Aires: Biblos; 2009.

18. Testa D. ¡S.O.S vacunas! Tensiones entre Estado y sociedad civil (1957-1971). En: Biernat C, Ramacciotti K, editoras. Políticas sociales, entre demandas y resistencias: Argentina, 19301970. Buenos Aires: Biblos; 2012.

19. Fitte M. Organización de los servicios para la asistencia del paralítico: Comunicación a la Sociedad Argentina de Pediatría, Sesión 13 de diciembre. Archivos Argentinos de Pediatría. 1939;11(2):129-146. [Material de Archivo]. Localizado en: Buenos Aires: Biblioteca Central Juan José Montes de Oca, Facultad de Medicina, Universidad de Buenos Aires.

20. Fitte M. Antecedentes, títulos y trabajos: Concurso de Profesor Adjunto de Ortopedia. Buenos Aires: Establecimiento Tipográfico de A. Guidi Buffarini; 1938. [Material de Archivo]. Localizado en: Buenos Aires: Biblioteca Nacional. 
21. Fitte M, Masucci A. Tratamiento de las secuelas de la poliomielitis. La Prensa Médica Argentina. 1938;XXV(50):2345- 2352. [Material de Archivo]. Localizado en: Buenos Aires: Biblioteca Central Juan José Montes de Oca, Facultad de Medicina, Universidad de Buenos Aires.

22. Fitte M. Asistencia social en la parálisis infantil. En: Relatos oficiales, contribuciones y discusiones de la Segunda Conferencia para el Bienestar del Lisiado. Buenos Aires: Asociación de Ayuda y Orientación al Inválido; 1946. p. 9194. [Material de Archivo]. Localizado en: Buenos Aires: Biblioteca Central Juan José Montes de Oca, Facultad de Medicina, Universidad de Buenos Aires.

23. Castro B. Las visitas domiciliarias en Colombia: Del trabajo voluntario a su profesionalización. En: Eraso Y, editora. Mujeres y asistencia social en Latinoamérica, siglos XIX y XX. Córdoba: Alción; 2009.

24. Lorenzo MF. Que sepa coser, que sepa bordar, que sepa abrir la puerta para ir a la Universidad. [Tesis de doctorado]. Buenos Aires: Universidad de San Martín; 2009.

25. Ciafardo E. Caridad y control social: Las sociedades de beneficencia en la ciudad de Buenos Aires, 1880-1920. Tesis de maestría, FLACSO, 1990.

26. Ramacciotti K, Valobra A. Profesión, vocación y lealtad en la enfermería peronista. En: Barry C, Ramacciotti K, Valobra A, editores. La Fundación Eva Perón y las mujeres: entre la provocación y la inclusión. Buenos Aires: Biblos; 2008.
27. Testa D. Poliomielitis: La "herencia maldita" y la esperanza de la rehabilitación: La epidemia de 1956 en la ciudad de Buenos Aires. Intersticios. 2011;5(2):309-323.

28. Carrillo R. Discurso Inaugural. En: Relatos oficiales, contribuciones y discusiones de la Segunda Conferencia para el Bienestar del Lisiado. Buenos Aires: Asociación de Ayuda y Orientación al Inválido; 1946. p. 20-25. [Material de Archivo]. Localizado en: Buenos Aires: Biblioteca Central Juan José Montes de Oca, Facultad de Medicina, Universidad de Buenos Aires.

29. Gamboa M. Discurso del Presidente del Comité Organizador de Segunda Conferencia para el Bienestar del Lisiado. En: Relatos oficiales, contribuciones y discusiones de la Segunda Conferencia para el Bienestar del Lisiado. Buenos Aires: Asociación de Ayuda y Orientación al Inválido; octubre de 1946. p. 36-40. [Material de Archivo]. Localizado en: Buenos Aires: Biblioteca Central Juan José Montes de Oca, Facultad de Medicina, Universidad de Buenos Aires.

30. Albert F. El Estado y la sociedad ante la invalidez del poliomielítico. En: Relatos oficiales, contribuciones y discusiones de la Segunda Conferencia para el Bienestar del Lisiado. Buenos Aires: Asociación de Ayuda y Orientación al Inválido; octubre de 1946. p. 95-10. [Material de Archivo]. Localizado en: Buenos Aires: Biblioteca Central Juan José Montes de Oca, Facultad de Medicina, Universidad de Buenos Aires.

\section{FORMA DE CITAR}

Testa DE. La lucha contra la poliomielitis: una alianza médico-social, Buenos Aires, 1943. Salud Colectiva. 2012;8(3):299-314.

Recibido el 23 de abril de 2012

Versión final presentada el 27 de junio de 2012

Aprobado el 8 de septiembre de 2012 